\title{
Anabases
}

ANABASES Traditions et réceptions de l'Antiquité

\section{Ubi tu Gaius, ibi ego Gaia. Enjeux historiographiques du mariage romain}

Sabine Armani

\section{OpenEdition}

1 Journals

Édition électronique

URL : http://journals.openedition.org/anabases/5445

DOI : 10.4000/anabases.5445

ISSN : 2256-9421

Éditeur

E.R.A.S.M.E.

Édition imprimée

Date de publication : 20 octobre 2015

Pagination : 63-84

ISSN : 1774-4296

\section{Référence électronique}

Sabine Armani, « Ubi tu Gaius, ibi ego Gaia. Enjeux historiographiques du mariage romain », Anabases

[En ligne], 22 | 2015, mis en ligne le 20 octobre 2018, consulté le 20 octobre 2019. URL : http:// journals.openedition.org/anabases/5445; DOI : 10.4000/anabases.5445 


\section{Ubi tu Gaius, ibi ego Gaia. Enjeux historiographiques du mariage romain}

Sabine Armani

e titre de la communication fait sienne la célèbre formule nuptiale traduite de Plutarque ${ }^{1}$ pour attirer d'emblée l'attention non seulement sur la chronologie du dossier traité ici qui ne prendra en compte ni le mariage chrétien ni la période tardive, qui a fait l'objet d'un récent état des lieux ${ }^{2}$, mais aussi sur la complexité de la question à aborder. Cette formule rituelle en est la première illustration. Elle est en effet traditionnellement associée à tort au rite nuptial en général alors que, semble-t-il, elle n'était prononcée qu'à l'occasion d'une procédure particulière, sur laquelle d'ailleurs il n'y a pas forcément consensus, la confarrea$t_{i o}{ }^{3}$ ou la coemptio ${ }^{4}$, que l'on évoquera plus loin. Plutôt que de traiter un dossier particulier (beaucoup de thèmes comme l'inceste, l'endogamie ou l'exogamie ont fait dernièrement l'objet de mises au point), l'on reviendra sur un siècle et demi d'historiographie relative au mariage, compris à la fois dans son acception cérémonielle et festive (les noces proprement dites) et institutionnelle (l'état marital

1 La formulation Vbi tu Gaius, ibi ego Gaia, à laquelle font indirectement allusion Cicéron, pro Murena, 27; Quintilien, de Institutione oratoria, I, 7, 28 et P. Festus, 95 a été traduite en latin d'après le témoignage de Plutarque, Questions romaines, 30.

2 Ch. Badel, Ch. Settipani (éd.), Les stratégies familiales dans l'Antiquité tardive, Paris, 2012.

3 C. FAYER, La familia romana. Aspetti giuridici ed antiquari. Sponsalia, matrimonio, dote. Parte seconda, Rome, 2005, p. 234 .

4 D. Gourevitch, M., Th. Raepsaet-Charlier, La femme dans la Rome antique, Paris, 20oi, p. 99 . 
qui résulte de la célébration de l'union). Le choix de lier ces deux aspects est parti d'un constat qui s'est naturellement établi au fur et à mesure de l'avancement des lectures. Il m'est apparu qu'une question était sous-jacente à la plupart des travaux relatifs aux pratiques matrimoniales: le mariage romain avait-il une base légale 5 ? Pour preuve, cette interrogation fait encore l'objet d'une discussion dans le dernier ouvrage consacré à la question, intitulé The Roman Wedding de Karen Hersch ${ }^{6}$. L'auteur, professeur à l'université de Temple, y apporte une réponse partagée: “à la fois oui et non » écrit-elle, sans pouvoir définitivement trancher. Même si la question n'a jamais été posée aussi ouvertement, il me semble que les positions de l'historiographie s'inscrivent de part et d'autre de cette ligne de partage: le mariage romain avait-il des aspects légaux ou non? La difficulté de se positionner ou la radicalité des options tiennent vraisemblablement à l'ambiguïté du vocabulaire, au type de mariage auquel on se réfère ainsi qu'à la fausse proximité que l'on a longtemps cru entretenir avec la famille romaine en général et le mariage en particulier' . L'intérêt croissant pour les provinces et les provinciaux a également provoqué un glissement du regard de l'historien, des noces proprement dites, dont il faut bien dire que l'on ne sait rien en dehors de Rome et de l'Italie, à l'étude de l'état qui en résulte, pour lequel les sources épigraphiques provinciales offrent un formidable réservoir. C'est pourquoi, après avoir examiné la littérature scientifique consacrée à la cérémonie proprement dite et aux procédures d'acquisition de la manus, c'est-à-dire l'autorité, sur l'épouse qui conditionnent en partie le rituel suivi, nous évoquerons la place croissante, ces dernières années, dans la recherche historique de l'étude de la vie conjugale, étude rendue possible par la prise en considération de nouveaux témoignages. La troisième et dernière partie fera le point sur les nouvelles pistes et leurs éventuels apports.

5 D’un côté, M. Corbier, «Famille et parenté: caractères originaux de la société romaine $\left(\mathrm{I}^{\mathrm{er}}\right.$ siècle av. J.-C.--III ${ }^{\mathrm{e}}$ siècle ap. J.-C.) », in A. Supiot (textes réunis par), Tisser le lien social. Florilège de dix années de conférences à la Maison des sciences de l'homme Ange-Guépin, Paris, 2004, p. 75: «... le mariage lui-même... reste une affaire privée, qui n’est soumise à aucune autorisation préalable, à aucune sanction officielle, à aucun enregistrement public. Ainsi le censeur ne faisait que noter, ex post, la situation qui lui était déclarée par les intéressés. » De l'autre, le titre évocateur de l'ouvrage du juriste G. Lind, Common Law Marriage. A legal institution for cohabitation, Oxford, 2008.

6 K. K. Hersch, The Roman Wedding. Ritual and Meaning in Antiquity, Cambridge, 20I0, p. 9: Was the wedding a legal act? The answer must be "yes and no".

7 Corbier, Famille et parenté, p. 73 : “La famille romaine est à la fois très proche et très éloignée de nous. » 


\section{La définition d'un premier champ d'étude: étude religieuse et symbolique du mariage}

\section{Le temps des pionniers}

Il n'est pas anodin de relever que le livre d'Augustus Rossbach, Untersuchungen über die römische Ehe, première somme sur le mariage romain publiée en I853, consacrait les deux premiers tiers de ses pages à des considérations juridiques avant d'accorder une place de choix, dans la dernière partie du livre, aux rites du mariage romain décrits en grande partie comme des rites agraires en raison de l'analogie que faisaient les Anciens entre mariage et travail des champs selon une image classique. La double nature du mariage romain, à la fois acte juridique et fête religieuse remontant à la plus haute Antiquité, à l'époque où Rome était encore une société pastorale, était alors posée comme allant de soi. A. Rossbach y faisait la recension détaillée de ces rites pastoraux, c'est d'ailleurs précise N. Boels Janssen, la vocation essentielle du livre ${ }^{8}$. La monumentalité de la thèse d'A. Rossbach a eu pour conséquence d'assécher un temps les études sur le mariage romain en empêchant le renouvellement des approches. Ses successeurs parmi lesquels Friedländer $^{9}$, Marquardt ${ }^{10}$, Blümmer ${ }^{11}$, Wissowa ${ }^{12}$ s'étaient surtout contentés de revenir de manière périphérique sur la question en proposant ici ou là quelques corrections ou interprétations marginales dans des ouvrages plus largement consacrés à la vie quotidienne ou à la religion romaine. À ce stade de l'analyse, je ne résiste pas à l'envie de verser au dossier l'existence d'un opuscule, déniché par hasard, que l'on doit au latiniste René Pichon, intitulé précisément Le mariage religieux à Rome et publié en igı. L'auteur y affiche un parti pris essentialiste dès l'introduction: le mariage romain est un acte purement religieux. Il faut sans doute remettre cette thèse dans le contexte de sa publication. À mots couverts dans son introduction, l'auteur établit ce qu'il croit être la nature profonde de l'institution maritale, “à l'heure, écrit-il, où le mariage subit l'assaut de discussions si nombreuses et si hardiment passionnées, il serait très intéressant de bien connaître les origines

8 N. Boels Janssen, La vie religieuse des matrones dans la Rome archaïque, Rome, I99I (CEFR, I76), p. I37.

9 L. Friedländer, Darstellung auf der Sittengeschichte Roms, éd. rev. par G. Wissowa, Leipzig, I9I9-I92I.

10 J. Marquardt, Das Privatsleben der Römer, Leipzig, i886 (Handbuchder römischen Alterthümer 7), traduit en français sous le titre La vie privée des Romains, Paris, I892-I893.

11 H. Blümmer, Die römischen Privataltertümer, Munich, IgII.

12 G. Wissowa, Religion und Kultus der Römer, Munich, IgI2 (2 éd.). 
d'une institution qui fait l'objet de tant de controverses ${ }^{13}$ ». Dans son illustration du mariage religieux, l'auteur n'hésite d'ailleurs pas à établir une hiérarchisation, non attestée par ailleurs, entre les différents types de mariage romain, conférant au rite de la confarreatio, ancienneté chronologique et supériorité sociale sur les deux autres procédures de mariage, celle par achat, la coemptio et celle par usus. Si ce qui ressemble à une instrumentalisation du mariage romain n'a pas fait date dans la production scientifique relative à la question maritale, en revanche le développement de l'anthropologie comparée allait bientôt permettre l'exploration de nouvelles pistes. L'ethnologue français Arnold Van Gennep proposa, dès I9og, une nouvelle lecture de la cérémonie nuptiale, fondée sur les rites de passage que l'étude des folklores érigée au rang de science, l'avait amené à identifier. Les comparaisons ethnographiques qu'il établissait l'avaient conduit à se familiariser avec le rituel nuptial romain, ce qui explique d'ailleurs que c'est à lui que sera confiée la traduction française de l'ouvrage de l'anthropologue Edward Westermarck parue en six tomes entre I935 et I945 dont le titre évocateur en français l'Histoire du mariage assura la diffusion des théories du savant finlandais sur l'exogamie et l'inceste. À propos du mariage romain, Arnold van Gennep dressait, dès Igog, une liste complète de ses rites dont il soulignait la valeur initiatique, parmi lesquels on retiendra, par exemple, la coiffure de la mariée dont le passage des cheveux flottants de la jeune fille aux cheveux retenus en tresses, les seni crines, fut jugé par lui caractéristique des rites de passage. Les progrès enregistrés par le développement de l'anthropologie et du droit comparés allaient bientôt servir de puissant stimulant capable de susciter de nouvelles études sur le mariage alimentées par ce nouveau regard croisé qu'on voit également s'exercer dans le livre de Madeleine Rage-Brocard, Rites de mariage: la deductio in domum mariti, publié à Paris en I934 ou dans celui de Percy Corbett paru quatre ans plus tôt. Dans son ouvrage, The Roman Law of Marriage ${ }^{14}$, le juriste anglo-saxon défendait la position suivante qui fut longtemps de mise chez les historiens du droit: “From the legal point of view, marriage in the classical period of Roman Law is almost, if

13 R. Pichon, Le mariage religieux à Rome, Paris, IgIo, p.3. Il poursuit: “ Or, ces origines, c'est à Rome qu'il faut les chercher. Qu'on l'envisage comme un contrat juridique ou comme une union morale, le mariage moderne est doublement latin... Par le Code civil comme par l'Église chrétienne, notre mariage vient de Rome; il plonge ses lointaines et robustes racines dans le sol latin. C'est ce qui donne à l'étude du mariage romain un intérêt tout particulier, actuel en quelque sorte. "Il faut relier ces prises de position au contexte anticlérical du début du xxe siècle qui culmina avec, en Igo5, la loi de séparation entre l'Église et l'État. Les défenseurs du mariage religieux avaient certainement dans leur viseur la loi sur le divorce de i884.

14 P. E. Corbett, The Roman Law of Marriage, Oxford, I93o. 
not entirely, a formless transaction ${ }^{15}$. „ On jugeait en effet qu'aucun acte dans la cérémonie du mariage - ni l'échange des consentements par la simple formule Spondesne? Spondeo aussi peu solennelle que celle servant à prononcer le divorce (tuas res tibi habeto), ni la signature du contrat fixant la dot en amont de la cérémonie, ni la consommation du mariage qui ne rencontre pas dans les sources littéraires le même écho qu'aux époques médiévales et modernes, mais qui ne doit pas être non plus minorée comme en témoigne dans l'épigraphie l'idéal de l'épouse uniuira (celle qui n'a connu qu'un seul homme) - n'était suffisamment saillant pour permettre d'affirmer qu'il marquait le début de la vie maritale du couple qui avait d'ailleurs pu se former avant les noces lorsque la fiancée, trop jeune, n’avait pas pu être épousée dans les formes. Les juristes s'appuyaient sur une réflexion d'Ulpien qui associait union et affectio maritalis, notion étrangère au droit, mais qui dans le Digeste concernait la poursuite du lien conjugal en cas d'absence prolongée de l'un des deux conjoints. Il est vrai que les mariages parodiques, ou non selon les analyses, auxquels se livrèrent, comme nous l'apprend Tacite, Messaline et Néron ${ }^{16}$ donnaient également en apparence raison à la position des juristes qui déniaient toute valeur légale à la cérémonie nuptiale.

\section{La « révolution» dumézilienne}

À la même époque et parallèlement se produisit un tournant dans l'interprétation symbolique du mariage romain dont les travaux de Georges Dumézil rendent le mieux compte. L'auteur de L'héritage indo-européen à Rome, publié en I949, nous donne une description vivante de la maturation de ses travaux dans l'introduction à ses Mariages indo-européens, paru en I979. Au printemps I943, en pleine Occupation, à l'occasion d'une réunion chez un collègue spécialiste du droit romain de la section des Sciences religieuses de l'École des Hautes Études, G.Dumézil présenta ses dernières réflexions dans lesquelles il indiquait qu'“...il devenait naturel de chercher si les concordances de détail remarquées depuis longtemps entre l'Inde et Rome en matière de mariage n'étaient pas des fragments cohérents d'une concordance plus générale, attestant l'existence d'une théorie déjà indo-européenne de cette partie du droit ${ }^{17}$ ». Si l'on met de côté le mythe fondateur du rapt des Sabines renvoyant à une forme de mariage archaïque sans consentement mutuel qui rappelle l'une des formes du mariage indien,

15 Conbett, Law ofMarriage, p. 67, cité par Hersch, Wedding, p.5i.

16 Tacite, Annales, I5, 37. Certains historiens ont assuré que le mariage de Messaline avec son amant était valide étant donné l'absence de formalisme du divorce romain.

17 G. Dumézil, Mariages indo-européens, suivi de Quinze Questions romaines, Paris, I979, p. IO. 
mais qui fonctionnerait aussi, l'on y reviendra, comme une synthèse de toutes les formes du mariage romain, coexistaient à Rome trois types d'unions ou plutôt trois procédures d'acquisiton de la manus sur l'épouse conformément au modèle dumézilien tripartite: la confarreatio tirant son nom de la célébration religieuse au cours de laquelle avait lieu la consommation rituelle d'un gâteau de farine d'épeautre et qui ne survit plus que de manière résiduelle à l'époque historique pour garantir la validité du mariage entre le flamine et la flaminique de Jupiter; la coemptio, achat symbolique par le mari, de sa femme qui se contente, comme l'a bien montré en son temps A. Rossbach, de signaler son consentement. C'est le volet économique du tableau trifonctionnel. Mais dans ces deux modes de mariage, la manus sur l'épouse est acquise immédiatement par la famille du mari. Vient enfin le mariage par la procédure de l'usus qui ne requiert aucune cérémonie particulière puisque c'est la pratique, c'est-à-dire la cohabitation du couple qui établit la vie maritale et l'usage qui assure l'acquisition différée de la manus. G. Dumézil exprime la dette intellectuelle qu'il a contractée auprès de ses collègues juristes qui l'ont dès le séminaire de 1943 mis sur la piste de la "deuxième fonction », la fonction guerrière, a priori invalidée par l'absence, dans le mariage par usus de toute trace de violence ${ }^{18}$. C'est par rapprochement entre les droits romain et indien, non pas avec le rapt des Sabines, mais avec l'un des huit types d'union indiens, le mariage Gāndharva qui considère l'union secrète par simple consentement mutuel des deux partenaires de la classe des kşatriya, celle des guerriers, comme une alliance valable aux yeux de la loi que le mariage par usus trouve sa place dans le schéma trifonctionnel. Il repose sur l'autonomie des contractants, notamment de la femme qui peut rompre la procédure acquisitive de la manus par l'usurpatio trinoctii, la séparation trois nuits de suite de son mari. Reste le mythe étiologique du rapt des Sabines, dans lequel certains avaient voulu voir une quatrième forme de mariage,

18 DumÉzil, Mariages, p.I7: “Le problème qu'annonce ce titre a été «vu » pour la première fois, au printemps de I943, par un spécialiste du droit romain que son œuvre antérieure ne disposait pourtant pas à lui prêter attention. C'est une longue histoire. »; p. I9: “Pierre Noailles était présent. Je ne le connaissais pas et je fus surpris de la chaleur de son approbation. Plus surpris encore quand je l'entendis proposer une extension de ma thèse à un domaine dont je ne m'étais pas occupé: le droit. [...] Noailles n'hésita pas à évoquer le principe de l'action guerrière - usage violent de la force - pour compléter, au niveau de l'usus, le tableau trifonctionnel. De la part de Noailles, c'était une conversion.»; p.29: “Je ne l'aurais [l'argumentation] cependant pas proposée à un plus large public si je n'avais bénéficié, ces dernières années, de véritables leçons particulières de droit romain qu'a bien voulu me donner mon éminent collègue $\mathrm{M}$. André Magdelain: se plaçant par hypothèse dans mon interprétation, il l'a armée de son savoir, de sa critique et de son sens juridique et, pour tout dire d'un mot, transfigurée. Si j'en assume la responsabilité, c'est lui qui en a le mérite. » 
tombée en désuétude, le mariage par enlèvement. G. Dumézil le considère comme le condensé de toutes les formes de mariage où il reconnaît les formes en gestation de la confarreatio par l'intervention de Romulus, l'usus par transformation du rapt en consentement réciproque, la coemptio, par l'acceptation finale des Sabins de donner leurs filles ${ }^{19}$.

\section{Les premières critiques}

Les réserves soulevées par le système dumézilien en général n'ont pas épargné le modèle trifonctionnel du mariage. On en trouve l'écho dans le second volume du triptyque de Carla Fayer, consacré aux fiançailles, au mariage et à la dot ${ }^{20}$. La juriste italienne y montre que, contrairement à l'opinion commune qui a encore cours, le mariage romain cum manu, scellé par l'une des formes d'acquisition de la manus sur l'épouse, n'aurait pas progressivement cédé sa place, à partir de la période médio-républicaine, à un nouveau type de mariage, le mariage sine manu, dans lequel la femme restait sous la dépendance juridique de son père jusqu'à la mort de ce dernier qui la hissait au rang de sui iuris. Carla Fayer dissocie à juste titre me semble-t-il mariage et dispositions juridiques concernant le statut de l'épouse. Rome n'aurait jamais connu qu'un seul type de mariage, un mariage libre qui unissait à égalité deux partenaires. C'était son association, ou non, avec la procédure de la confarreatio, de la coemptio ou de l'usus qui modifiait le statut de l'un des deux conjoints, en l'occurrence l'épouse. Il n'existait donc pas deux formes de mariage cum ou sine manu, dont la dernière aurait fini par s'imposer sous l'Empire, mais seulement une uxor soumise ou non à la manus de sa belle-famille. D'ailleurs les sources ne nous parlent que de manus appliquée à l'épouse sans référence explicite au mariage. C'est par effet métonymique que la manus a été associée par les modernes à l'union ${ }^{21}$. Ce changement de perspective contribue à modifier substantiellement la nature du mariage romain et ce que l'on croyait connaître de son évolution. Les modes d'acquisition de la manus seraient donc des actes indépendants de la cérémonie du mariage. Or, si la confarreatio, la coemptio et l'usus constituaient les fossiles, appliqués au mariage, de la trifonctionnalité indo-européenne, on comprend mal que ces trois opérations aient été dissociées de la cérémonie du

19 Dumézil, Mariages, p. 76 et suivantes: “Ainsi, dans le sillage du rapt, nous voyons se créer la confarreatio, mûrir l'usus, s'esquisser la coemptio.»

20 FAYER, La familia romana, p I85-325 (La conventio in manum).

21 Gaius dans ses Institutiones (I, IIO-III) parle d'uxor in manu mariti, abrégé en uxor in manu dont le statut serait antérieur à la Loi des XII Tables (M.Bretin-Chabrol, L'arbre et la lignée. Métaphores végétales de la filiation et de l'alliance en latin classique, Grenoble, 20I2, p.I98). 
mariage qui pouvait se tenir indépendamment de l'une de ces procédures. De plus, Carla Fayer rappelle que la confarreatio est progressivement tombée en désuétude ce qui est difficilement compatible avec les théories duméziliennes, désuétude que les dispositions du sénatus-consulte de 23 p. C. essayaient justement de combattre afin d'assurer la continuité du recrutement des couples flaminaux de Jupiter. Elle évoque également l'élargissement de la coemptio à d'autres domaines que le seul mariage. Le travail de Nicole Boels Janssen, publié en r99ı, s'inscrit en revanche dans la lignée de ses grands devanciers: les références à A.Rossbach, sur la dimension agraire des mythes ${ }^{22}$, à van Gennep sur le caractère initiatique de certains cérémoniaux et à Dumézil, sur l'essence religieuse du mariage romain, le rattachent tout naturellement à eux. Cependant, son travail ne considère plus le mariage comme l'alpha et l'oméga de la vie matronale, mais comme un élément, certes nodal, qui s'inscrit néanmoins dans une chaîne de pratiques rituelles qui rythment la vie de la citoyenne romaine depuis l'enfance. La méthode suivie consiste principalement à retrouver derrière le foisonnement des mythes étiologiques (le rapt fondateur des Sabines, le meurtre de Camille, etc.), la sédimentation des nombreux rituels et l'empilement des interprétations étymologiques des lexicographes « aux rapprochements - écrit-elle - trop évidents parfois », la signification symbolique des usages dont le sens s'est totalement perdu à l'époque historique et qui seraient répétés de manière machinale. Les lectures juridiques de la gestuelle nuptiale sont donc a priori écartées. Les différents moments de la cérémonie matrimoniale auraient donc vocation à demeurer privés, comme la prise des auspices. Le seul acte juridique identifié comme tel ${ }^{23}$ - la signature du contrat de mariage qui se fait devant témoins - demande à être ratifié par un geste religieux, la dextrarum iunctio, l'union des deux mains droites des époux, symbole de leur concordia et de leur engagement réciproque.

À partir des années soixante-dix, sous l'influence des enquêtes ethnologiques et de la sociologie, il est progressivement apparu que l'analyse du mariage ne se résumait pas seulement à l'étude des rituels de la noce, mais devait prendre en compte aussi les aspects de la vie conjugale. Cette prise de conscience s'est produite au rythme de l'intégration de nouveaux documents qui acquéraient progressivement un droit de cité, égal à celui des témoignages littéraires, je veux parler des sources épigraphiques et papyrologiques.

22 C'est d'ailleurs au livre de N.Boels Janssen que nous devons d'avoir pu prendre connaissance du contenu de l'ouvrage d'A. Rossbach introuvable en France.

23 Boels Janssen, La vie religieuse, p. I39: “Mais le premier acte du mariage lui-même était la signature du contrat de mariage, en présence de témoins. Malgré son importance pour l'esprit essentiellement juridique des Romains, nous ne nous y attarderons pas car il n'a rien de religieux et n'est qu'un préliminaire juridique indispensable au rituel nuptial. » 


\section{Mariage et épigraphie: nouvelles approches}

\section{L’influence des sciences sociales}

Dans la lignée de la pensée lévi-straussienne, les historiens de la parenté romaine se sont d'abord employés à débusquer les invariants culturels supposés marqueurs, au-delà des différences locales, de structures élémentaires communes à l'humanité tout entière. Il est naturel que les effets de cette méthode fondée sur le primat de la linguistique aient rencontré un écho particulier chez les spécialistes de la langue latine. De ce point de vue, la terminologie latine de la parenté offrait un réservoir encore peu exploré, susceptible de remplacer les données que les ethnologues pouvaient recueillir sur le terrain auprès des populations qui les accueillaient. En 1978, Philippe Moreau consacrait un article à la terminologie latine de la parenté et de l'alliance: comme le titre l'indiquait sans ambiguités ${ }^{24}$, il y livrait les résultats que les méthodes de la linguistique anthropologique appliquée à sa discipline autorisaient alors à obtenir. Dans l'approche lévi-straussienne, penser l'inceste était inséparable de la compréhension des mécanismes du mariage qui se fonde sur l'échange des femmes, rendu possible par l'interdiction d'épouser sa sœur. Rien d'étonnant alors à ce que les historiens de la parenté romaine, formés à la lecture des anthropologues français, aient parallèlement orienté leurs recherches vers les prohibitions matrimoniales d'autant plus que la législation romaine en la matière a connu des évolutions contradictoires passant d'une levée progressive des restrictions à leur extension, rendue plus drastique encore à partir de Dioclétien où certains interdits matrimoniaux s'appliquaient désormais à des degrés jusqu'ici épargnés ${ }^{25}$. Ces mouvements opposés de la législation trouvèrent leur archéologue en la personne de M.Bettini qui proposa, en se fondant sur le périmètre d'application du ius osculi, cet antique droit au baiser des femmes sur certains de leurs parents, d'identifier, à l'époque archaïque, le dernier cercle de conjoints interdits à ceux qui étaient parents au sixième degré26, ce qui correspond

24 Ph. Moreau, «La terminologie latine et indo-européenne de la parenté et le système de parenté et d'alliance à Rome „, REL 56 (I978), p.4I-53.

Le point dans Ph. Moreau, «Le mariage dans les degrés rapprochés. Le dossier romain ( ${ }^{\mathrm{er}}$ siècle av. J.-C.-III ${ }^{\mathrm{e}}$ siècle ap. J.-C.) ", in P. Bonte (dir.), Épouser au plus proche. Inceste, prohibitions et stratégies matrimoniales autour de la Méditerranée, Paris, 1994, p.59-78. Pour la période tardive, compléter avec D.Lhuillier-Martinetti, L’individu dans la famille à Rome au IV siècle d'après l'ouvre d'Ambroise de Milan, Rennes, 2008.

26 M. Bettini, “ Il divieto fino al " sesto grado » incluso nel matrimonio romano », in J. Andreau et H. Brunns (textes réunis et présentés par), Parenté et stratégies familiales dans l'Antiquité romaine. Actes de la table ronde des 2-4 octobre Ig86 (Paris, Maison des 
à nos cousins « au second degré». L'obligation faite encore au $\mathrm{II}^{\mathrm{e}} \mathrm{s}$. p. C. semble-t-il, aux femmes d'embrasser sur la bouche leurs parents mâles jusqu'aux cousins issus de cousins trouvait sa signification dans une ancienne prohibition matrimoniale qui touchait les cousins éloignés dont on déduit qu'elle fut levée avant 200 a. C., date de l'autorisation du mariage entre cousins germains signalée par une notation livienne ${ }^{27}$. Cette attention accrue aux interdits matrimoniaux allait cependant devoir tenir compte de deux objections. La première venait des sources ellesmêmes et notamment du dossier papyrologique égyptien qui révélait l'absence de normes universellement admises en matière d'échange avec la possibilité, non érigée en principe matrimonial cependant à la différence d'autres sociétés, de conclure une alliance avec sa sœur ou sa demi-sœur. Cette alliance était bien sûr interdite aux citoyens romains, mais tolérée chez les pérégrins - sujets de l'empire - et comme les recensements égyptiens l'indiquent, perdura jusqu'en 2 I2 p. C. K. Hopkins fut le premier à en instruire le dossier ${ }^{28}$ dont les derniers développements sont cependant récents tant il continue à susciter l'incompréhension d'un certain nombre de collègues qui essaient d'en diminuer la portée ${ }^{29}$. C'est à F. Héritier, élève et successeur de Cl.Lévi-Strauss au Collège de France, que l'on doit la seconde objection ${ }^{30}$. Celle-ci a également remis en cause la place centrale de la prohibition de l'alliance avec la sœur dans la théorie de l'échange par le constat de l'existence universelle de prohibitions matrimoniales pesant cette fois sur des parents appartenant pourtant au groupe exogamique d'Ego (beaux-frères, beauxparents, beaux-enfants, etc.). L'identification d'un deuxième cercle de parents tabous trouvait une explication symbolique dans l'interdiction de la circulation

sciences de l'homme), Rome, I99o, p. 27-52; M. Betтini, Affari difamiglia. La parentela nella letteratura e nella cultura antica, Bologne, 2009, chapitre IX « In vino stuprum. Ovvero, le donne romane che non bevono vino ", p. 239-258.

27 Tite-Live, Histoire romaine, XLII, 34.

28 K. Hopkins, “Le mariage frère-sœur en Égypte romaine», in Bonte, Épouser au plus proche, p. 79-III. Dans la note 2 de l'article, l'auteur précise que l'étude “ est la version revue et abrégée d'une précédente publication [«Brother-sister marriage in Roman Egypt », Comparative Studies in Society and History, 22 (I980), p.3o3-354] „ Présentation commode du dossier dans B. Legras, Hommes et femmes d'Égypte (IV s. av. n. è.-IV s. de n. è.). Droit, histoire, anthropologie, Paris, 2010, p. I83-192.

29 S. HüBnER, “Brother-Sister" Marriage in Roman Egypt: a Curiosity of Humankind or a Widespread Family Strategy? », JRS 97 (2007), p. II-49 qui propose de voir dans cette pratique l'adoption du gendre par son beau-père plutôt qu'un mariage entre frère et sœur. Contra, S. Remissen, W. Clarysse, “Incest or Adoption? Brother-Sister Marriage in Roman Egypt revisited », JRS 98 (2008), p.53-6r.

30 R. Deliege, Anthropologie de la parenté, Paris, I996, p.4I-42. Sur le parcours de Françoise Héritier, lire aussi son interview (Libération du 26 mars 2009). 
d'humeurs vitales communes ${ }^{31}$. Philippe Moreau a introduit la notion d'“inceste du deuxième type „ formulée par F. Héritier dans son analyse des interdits matrimoniaux. Il aborde également ce thème en I978 dans un article dont le titre apparaît avec le recul comme un véritable manifeste méthodologique convoquant les sources anciennes au même titre que les études menées sur le terrain par les ethnologues auprès des populations qu'ils étudient ${ }^{32}$. L'un des premiers plaidoyers de Cicéron - le pro Cluentio, discours dans lequel son client éponyme est accusé du meurtre de son beau-père - va donner à Philippe Moreau l'occasion de mettre sa méthode à l'épreuve. La ligne de défense de Cicéron consiste à discréditer la famille de la victime, à commencer par Sassia, la mère de Cluentius, qui avant de se remarier au défunt avait épousé en secondes noces son gendre ravi à propre sa fille. C'est l'immoralité du couple qui aurait poussé l'accusé à l'homicide. L'évocation de mariages entre collatéraux enrichit l'imbroglio familial que Philippe Moreau entreprend d'éclairer par l'identification du type de cousins impliqués dans l'alliance ${ }^{33}$. La question de l'inceste alimentera ensuite un ouvrage tiré de sa thèse d'état ${ }^{34}$. Le remariage de Sassia, la mère de Cluentius, avec son gendre n'avait pour autant pas fait l'objet de poursuites légales, ce qui a même suggéré que ce type d'alliance entre affins hétérostathmiques était encore autorisé à la fin de la République. Son interdiction postérieure serait, selon l'anthropologue Laurent Barry, qui s'appuie sur les travaux de Ph. Moreau et de P.Veyne ${ }^{35}$, le témoignage incontestable de “l'invention du couple ${ }^{36}$ ». L'assimilation de certains alliés à des conjoints prohibés aurait alors été le signe indubitable d'une révolution conjugale. L'essor de l'anthropologie a également largement influencé les travaux de Mireille

31 F. Heritier, Les deux sours et leur mère, Paris, I994.

32 Ph. Moreau, “Plutarque, Augustin, Lévi-Strauss: prohibition de l'inceste et mariage préférentiel dans la Rome primitive », $R B P h 56$ (1978), p. 4i-54.

33 Ph. Moreau, “Structures de parenté et d'alliance à Larinum d'après le Pro Cluentio », in Les bourgeoisies municipales italiennes aux II et $I^{e r}$ s. av. J.-C., Paris-Naples, I983 (Colloques internationaux du cNRs, 6o9), p.99-I23 complété par Ph.Moreau, “Patrimoines et successions à Larinum au i ${ }^{\mathrm{er}}$ s. av. J.-C. », RHDFE 64 (I986), p. I69-189.

$34 \mathrm{Ph}$. Moreau, Incestus et prohibitae nuptiae. Conception romaine de l'inceste et histoire des prohibitions matrimoniales pour cause de parenté dans la Rome ancienne, Paris, 2002 (Collection d'Études anciennes, 26).

35 P. Veyne, “La famille et l'amour sous le Haut-Empire romain », Annales ESC 33, I (I978), p.35-63.

36 L. BARry, La parenté, Paris, 2008, p.496-497: “Si le mariage entre adfines de générations différentes - celui, par exemple, d'un beau-père et d'une bru ou d'une marâtre et d'un beau-fils - était déjà semble-t-il désapprouvé à la fin de la République, il ne relevait alors que de la catégorie du stuprum, de la débauche [...] ou, dans le pire des cas, de celle d'adulterium, de l'adultère. » 
Corbier qui en a importé certains concepts et qui convoque souvent en prélude à une étude la figure tutélaire de Cl. Lévi-Strauss ${ }^{37}$. Elle fut également la première à s'intéresser à la «double relation de parenté » entre époux et cousins par exemple, relations plurielles dont l'analyse se déploie dans plusieurs travaux. Entamé en I990 dans un article intitulé “Construire sa parenté à Rome ${ }^{38}$ », l'inventaire des occurrences épigraphiques commençait par distinguer les attestations explicites de double lien de parenté par l'indication de deux termes, l'un appartenant à la parenté par le sang (cognatique), l'autre à l'alliance (adfinitas) ${ }^{39}$, des cas implicites seulement décelables par l'onomastique des époux homonymes ${ }^{40}$. Dans le dernier volet de la série des colloques australiens sur la parenté, inaugurée en I98I par Beryl Rawson ${ }^{41}$, elle produisit une synthèse régionale et provinciale qui lui permettait d'approfondir le riche cas africain par le repérage systématique, dans les sources littéraires et épigraphiques, de mariages échappant aux règles de l'exogamie traditionnelle, parmi lesquels ceux que les anthropologues désignent comme remarquables parce qu'ils impliquent un «renchaînement d'alliances ». Le lévirat qui consiste à épouser la veuve de son frère, autorisé à Rome jusqu'au III $^{\mathrm{e}}$ siècle de

37 Par exemple, Corbier, Famille et parenté, p. 74: “C'est sur cet écart, fait de distance et de familiarité, que je voudrais porter le regard "éloigné" de l'anthropologue autant que de l'historien.»

38 M. Corbier, “ Construire sa parenté à Rome », $R H 575$ (I990), p.3-36.

39 Corbier, Construire sa parenté, p. 9: «Les inscriptions qui font apparaître un double lien de parenté méritent évidemment une attention toute particulière. Ainsi l'inscription funéraire par laquelle un gener honore son patruus témoigne-t-elle vraisemblablement d'un mariage entre cousins parallèles patrilatéraux (CIL, VI, I7534: à Rome; gendre et beau-père y portent bien le même gentilice, Fabius). Le même type de mariage est indiqué par l'expression uxor eadem soror patr(u)elis (CIL, VI, 8409: milieu des esclaves et affranchis impériaux). La désignation de la même femme comme socrus et amita par sa nièce et belle-fille homonyme révèle un mariage entre cousins croisés (RIC, II, 5o: à Aeso, en Tarraconaise). Présenter sa conjointe comme consobrina eademque uxor signifie que l'on a épousé une cousine germaine (ILS, 9I5 : à Histonium, en Italie)... ».

40 Corbier, Construire sa parenté, p. Io: “À elle seule l'onomastique peut témoigner d'un mariage entre proches parents. Tout devient affaire de milieu social. Si statistiquement les conjoints porteurs du même nom sont souvent les affranchis d'un même patron, ou le patron et son affranchie, la similitude des gentilices peut témoigner aussi d'un mariage entre cousins patrilatéraux ou tout au moins entre agnats. L'épouse qui porte pour cognomen le gentilice de son mari pourrait être sa cousine croisée patrilatérale...»

41 B. Rawson (éd.), The Family in Ancient Rome. New Perspectives, Londres-Sydney, I986; B. Rawson (éd.), Marriage, Divorce and Children in ancient Rome, Oxford-Canberra, I99I; B.Rawson, P.R. C. Weaver (éd.), The Roman Family: Status, Sentiment, Space, Oxford, I99I; M. George (éd.), The Roman Family in the Empire. Rome, Italy and Beyond, Oxford, 2005. 
notre ère, semble avoir rencontré dans l'Afrique romaine un écho particulier interprété par les historiens comme un vestige d'usages puniques. Le dialogue avec l'anthropologie comparée ne s'arrête pas là. Il avait commencé dans un premier article où l'auteur se proposait de confronter pratiques aristocratiques romaines et modernes de la famille ${ }^{42}$. Le tableau comparatiste sera ensuite complété d'un second volet qui prendra entre autres pour modèle les stratégies médiévales ${ }^{43}$. Le concept de “stratégies», emprunté aux sociologues ${ }^{44}$ qui l'avaient eux-mêmes élaboré pour contrer celui, vieillissant, des “structures », va parallèlement s'avérer fécond pour les romanistes que la parenté intéresse. Dans son introduction au récent colloque intitulé Les stratégies familiales dans l'Antiquité tardive, Christophe Badel $^{45}$ a en effet mis en évidence ce tournant pris par l'historiographie de la famille dans les années quatre-vingt qui consistait à s'intéresser à l'individu, puis au groupe (ici familial) pris dans une stratégie de l'alliance. Cette recherche se concrétisa dans la publication en rg9o de l'imposant volume Parenté et stratégies familiales dans l'Antiquité romaine ${ }^{46}$. Ch. Badel rappelle les discussions que la notion de stratégie suscita bientôt chez les historiens de la République et de l'Empire, dont certains contestèrent le bien-fondé de l'application à des familles dont on ignorait pour la plupart tout de la capacité à se projeter dans l'avenir ou l'éventail des choix de l'alliance. Il n'empêche. Depuis les années quatre-vingt, l'attention prêtée à la séparation des couples dans la société aristocratique romaine tient autant aux considérations d'une époque où le divorce s'est banalisé, qu'à l'intérêt nouveau suscité par l'exploration des possibilités offertes aux familles ou aux individus de se choisir des alliances plus juteuses ${ }^{47}$. D'un point de vue strictement historiographique, il n'est pas anodin de constater le glissement des objets d'étude

42 M. Corbier, “Les comportements familiaux de l'aristocratie romaine (II siècle av. J.-C.III $^{\mathrm{e}}$ siècle ap. J.-C.) "), in Andreau-Bruhns, Parenté et stratégies familiales, p.225-249; M. Corbier, "Parenté et pouvoir à Rome», in Ph. Genet (études réunies par), Rome et l'État moderne européen. Actes du colloque de Rome, janvier-février 2002, Rome, 2007 (CEFR, 377), p. I73-20I (dédié à Martin Heinzelmann). Corbier, Famille et parenté, p. 73-9i.

Sur les convergences des deux disciplines, N. Offenstadt, L'historiographie, Paris, 20II (esJ, 3933).

BADEL, Les stratégies familiales, p. V-XX.

Ouvrage cité aux notes 26 et 42.

M. Corbier, “Le divorce et l'adoption «en plus » , in Ch. Hamdoune (éd.), Vbique amici. Mélanges offerts à J.-M.Lassère, Montpellier, p.35ı-388; Conвıв, Famille et parenté, p. 78-8o. 
du remariage ${ }^{48}$ aux analyses qui s'intéressent désormais exclusivement à la dissolution des liens du mariage ${ }^{49}$.

\section{La domination des études quantitatives}

Sur la question du mariage, l'historiographie anglo-saxonne n'est pas en reste, mais elle s'est engagée sur d'autres voies. Sous l'influence d'historiens modernistes, allait naître le groupe de Cambridge ${ }^{50}$ fondé par le démographe comparatiste Peter Laslett, auteur de l'ouvrage devenu best-seller The World that we have lost: England before the Industrial Age (ig65), dans lequel il faisait voler en éclats quelques idées reçues sur la famille en affirmant notamment contre tous les modèles établis jusque-là la prééminence du modèle de la cellule familiale nucléaire dans l'Angleterre de l'époque moderne. Le groupe de Cambridge avait donné le coup d'envoi, dans le monde anglo-saxon d'abord, aux études de démographie historique. Les travaux de Keith Hopkins sur la démographie antique,

48 M. Humbert, Le remariage à Rome. Étude d'histoire juridique et sociale, Paris, I972.

49 Rawson, Marriage, Divorce; J. Evans Grubbs, Women and the Law in the Roman Empire. A Sourcebook on Marriage, Divorce and Widowhood, Londres, 2002.

50 M. GEorge, «Introduction », in GEorge (éd.), The Roman Family, p. I : « His volume follows the three Australian conferences begun by Beryl Rawson in I98I, a time when, quite coincidentally, a number of scholars in different parts of the mainly Anglo-American world were beginning to focus on the topic of the Roman family as a distinct theme in ancient social history. Research on family studies in the ancient world was in part the result of a growth of interest in the ig6os and ig7os in the history of the family among historians at large (e.g. Laslett, MacFarlane, Stone), and, for some, the growth of a very "scientific" approach to demographic studies, represented especially by the Cambridge Group for the History of Population and Social Structure. From these new historical methodologies emerged Keith Hopkins's pioneering application of demography to the ancient context, which opened up a new form of analysis that continues to thrive while remaining highly controversial; p.3: Definition, however, is not the only issue which has engaged historians of the family. The last generation of scholarship has focused on the component elements of family life that are regarded now and were regarded then as important: paternal power, family law, marriage patterns (including divorce and remarriage), the history of children and childbearing, the life course, old age, relations between family members (spouses, parents, and children), as well as between kin and non-kin members of the household. Instead of trying to contain the Roman family within a particular set of parameters, such studies have adumbrated the diverse elements of domestic life and considered their interplay. Rather than resolving the question of structure, they have complicated it by enhancing our understanding of the many dimensions of experience which fall within the category of "family life", but for which the issue of structure has only minor relevance.» 
inspirés par Moses Finley, s’y rattachaient explicitement. À la fois historien de l'Antiquité et sociologue, il est entre autres l'auteur d'une étude, "The Age of Roman Girls at Marriage», parue dans Population Studies, I8, p.3og-327, qui annonce les travaux postérieurs de R. Saller et B. Shaw. En effet, le tournant décisif fut donné par les analyses quantitatives de ces deux chercheurs qui, en s'appuyant sur l'étude systématique des épitaphes, déduisaient dans un article resté célèbre $^{51}$ que la famille romaine, sur le modèle de la famille anglaise des Lumières, était nucléaire ${ }^{52}$. Ces conclusions s'accompagnaient d'un constat développé dans un article de la même année, Close-Kin Marriage in Roman Society, paru dans la revue d'anthropologie (non d'Histoire) ${ }^{53}$, selon lequel le mariage conclu entre proches, qui caractérise les familles étendues, était très peu répandu, ceci en nette réaction aux thèses de l'anglais Jack Goody, spécialiste d'anthropologie comparée, qui en I983 publiait son ouvrage de référence The Development of the Family and Marriage in Europe dans lequel il datait la disparition de l'endogamie comme mode d'alliance généralisé, de l'avènement de l'empire chrétien ${ }^{54}$, mais aussi en réponse aux travaux de Y. Thomas, qui soulignaient la tendance à l'endogamie des familles romaines tant aristocratiques que populaires ${ }^{55}$. Ces travaux sur l'endogamie n'étaient pas isolés, mais s'inscrivaient dans une tradition ouverte depuis les années soixante, qui se fondaient en partie sur les progrès de la linguistique comparée dont les conclusions sont aujourd'hui critiquées. Dans son imposant Vocabulaire des institutions indo-européennes ${ }^{56}$, É. Benveniste avait par exemple avancé, pour expliquer la formation sur auus (le grand-père) du terme auunculus, qui désigne en latin l'oncle maternel, l'hypothèse d'un mariage préférentiel - dont on n'a pas la trace historique - entre cousins croisés (fils et fille d'un frère et d'une

51 R. P. Saller, B. D. Shaw, "Tombstones and Roman Family Relationships in the Principate: Civilians, Soldiers and Slaves », JRS 74 (I984), p. I24-ı56.

52 J. Edmondson, “Family Relations in Roman Lusitania », in George, The Roman Family, p. I87-188: «Saller and Shaw's main conclusion was that in civilian families in the Roman west funerary commemorations were made in the overwhelming majority of cases by members of the nuclear family: most commonly, wives commemorating husbands or vice versa, etc. »

Man, I9 (I984), p.432-444.

54 Moreau, Le mariage dans les degrés rapprochés, p. 66.

55 Y. Thomas, “Mariages endogamiques à Rome. Patrimoine, pouvoir et parenté depuis l'époque archaïque», RHDFE 58, 4 (1980), p.345-382. Sur ce débat, voir les éclairages récents sur l'Orient tardif de Ch.Freu, “Croissance de l'endogamie en Orient sous l'Antiquité tardive? L'analyse du cas égyptien ", in Badel-Settipani, Les stratégies familiales, p. I27-152 qui conteste la thèse d'un repli endogamique.

56 É. Benveniste, Le vocabulaire des institutions indo-européennes, t. I: économie, parenté, société, Paris, 1969. 
sœur) qui faisait du grand-père paternel de l'enfant du couple (l'Ego des anthropologues), l'oncle maternel de l'oncle maternel de ce dernier, son "petit grandpère» en quelque sorte ${ }^{57}$. Ceci n'empêcha cependant pas les résultats de R. Saller et B.Shaw de connaître un écho spectaculaire dans le monde anglo-saxon ${ }^{58}$, qui laissa au départ peu de place aux réserves émises par une partie de la communauté scientifique ${ }^{59}$. En France, mais pas seulement, Mireille Corbier ${ }^{60}$ et Philippe Moreau affichèrent bientôt leur scepticisme en remettant en cause la méthodologie même des travaux qui opposaient un peu vainement endogamie et exogamie alors que les deux pratiques avaient dû coexister à l'intérieur des familles. Sur les résultats incomplets de l'étude de Saller et Shaw parue dans Man qui ne tenaient compte que des cas de mariages entre cousins identifiables par une onomastique commune $^{61}$ (les cousins parallèles patrilatéraux, donc titulaires d'un gentilice identique), il a même été avancé que la situation particulière des États-Unis où les deux chercheurs exercent, qui interdisent ce type de mariage avaient pu influencer l'orientation des recherches. En ce qui concerne les milliers d'épitaphes

57 Reconstitution, d'après É. Benveniste, de l'hypothèse d'un mariage préférentiel entre cousins croisés qui expliquerait l'étymologie d'auunculus:

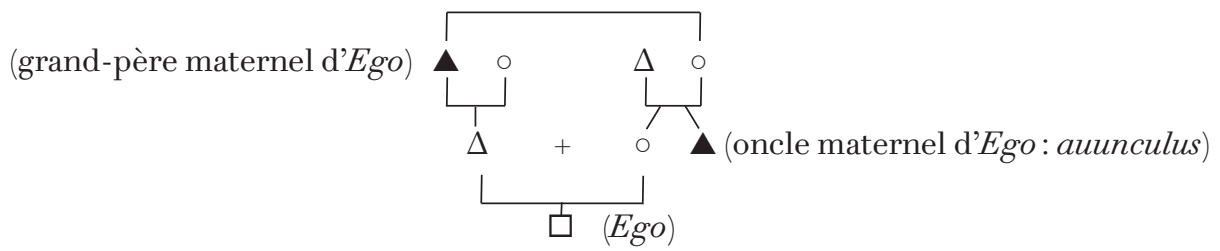

58 S. Dixon, The Roman Family, Baltimore, I992; J. F. GARDner, Women in Roman Law and Society, Londres-Sydney, 1986.

59 Voir Edmondson, Family Relations, p. I87, note I2.

60 M. Corbier, Epigraphie et parenté, in Y. Le Bohec, Y. Roman (éd.), Epigraphie et histoire: acquis et problèmes. Actes du Congrès de la SoPHAU, Lyon-Chambéry, 2I-23 mai Igg3, Lyon, I998, p.I2I; J.-U.Krause, “Familien- und Haushaltsstrukturen in spätantiken Gallien», Klio 72 (I99i), p.537-562; D.B.Martin, "The Construction of the Ancient Family: Methodological Considerations », JRS 86 (I996), p.40-6o; pour: B. Rawson, “The "Family" in the Ancient Mediterranean: Past, Present, Future », ZPE II7 (I997), p. 294-296.

61 M. Corbier, "Pour une pluralité des approches prosopographiques », MEFRM IOo (I988), p. I96-I97: “Il ne suffit pas de constater - fort justement d'ailleurs - que les centaines d'épitaphes de conjoints s'inscrivent en faux contre l'hypothèse d'un mariage préférentiel entre cousins parallèles patrilatéraux qu'il serait facile en effet de détecter, en raison des usages onomastiques romains, par l'identité du nom de l'époux et de l'épouse. Mais il faut dire aussi que l'observation ne suffit pas à écarter l'éventualité du mariage avec la cousine croisée ou avec une cousine au second degré (le $6^{\text {e }}$ ou $5^{\text {e }}$ degré de parenté romaine), c'est-à-dire issue de germains - qui ne portent pas le même nom. » 
dépouillées par Saller et Shaw, réalisées pour la plupart par des proches, elles ne renvoyaient en fait de la famille romaine qu'une image biaisée. Les épitaphes reflétaient ce devoir de piété familiale ${ }^{62}$, mais ne permettaient absolument pas de conclure que la famille romaine était nucléaire. La synthèse de Susan Treggiari, Roman Marriage. Iusti Coniuges from the Time of Cicero to the Time of Ulpian, publiée en I99I, mais dont les prémices remontent à la fin des années I970, a pourtant été largement influencée par les résultats de la méthode quantitative au moins dans l'interprétation des sources épigraphiques. Dans sa préface, l'auteur exprime la dette intellectuelle contractée auprès du groupe de Cambridge ${ }^{63}$. Il est d'ailleurs très intéressant d'observer que certains des aspects les plus discutés du mariage romain - concernant les modalités de passage de la future épouse d'une famille à l'autre (usus, confarreatio, coemptio) - n'ont droit ici qu'à une rapide présentation. Certains chapitres révèlent plus que d'autres encore les préoccupations alors dominantes dans l'historiographie: “Coniugalis Amor» ou encore le titre de la quatrième partie «Paterfamilias and Materfamilias» dont les conclusions sont directement inspirées des résultats statistiques liés à l'âge moyen au décès tirés des travaux de R. Saller et B. Shaw et de leurs conclusions sur la réalité de la patria potestas $^{64}$. En effet, dans une étude préliminaire ${ }^{65}$, R. Saller déduisait à la fois de l'âge au mariage des hommes - plus élevé que l'on ne s'y attendait - qu'il estimait en moyenne entre 25 et 30 ans grâce à l'intervention récurrente des épouses dans les épitaphes de leur mari à partir de 25 ans, et de la fréquente absence de la figure paternelle dans les épitaphes des enfants à partir de i8 ans, l'inefficience de la puissance paternelle en raison de l'impossibilité du titulaire, déjà décédé, à l'exercer. La fin du siècle passé enregistre les réserves de plus en plus nombreuses formulées par la communauté scientifique à l'encontre de la méthode quantitative. Jonathan Edmondson, pourtant disciple de la méthode Saller et Shaw, redéfinit le

62 M. Corbier, “Parenté et alliance dans le monde romain ( $\mathrm{II}^{\mathrm{e}}$ siècle a. C.-vi ${ }^{\mathrm{e}}$ siècle p. C.) ), in A. Bresson et al., Parenté et société dans le monde grec de l'Antiquité à l'Âge moderne. Colloque international Volos (Grèce), Ig-2I juin 2003, Bordeaux, 2006, p. 205.

63 P. IX : “Although epigraphic sources are not prominent in this book, I am also grateful to those who have explored the ways of using tomb inscriptions to answer questions of social history, scholars such as M. K. Hopkins, Beryl Rawson, Richard P. Saller, and Brent D. Shaw. »

64 R. Saller, B. Shaw, Patriarchy, Property and Death in the Roman Family, Cambridge, I994.

65 R. Saller, “Men's Age at Marriage and its Consequences in the Roman Family », $C P h 82$ (I987), p. 2I-34. 
périmètre de son application qu'il limite désormais à l'évaluation de l'intensité des relations familiales, non à la connaissance des structures de la parenté ${ }^{66}$.

\section{Le retour de la prosopographie}

Les années quatre-vingt sont également marquées, avec la découverte de nouveaux documents en Espagne - comme la loi municipale d'Irni traduite en français en $1986^{67}$ - par un regain des études consacrées au droit latin, dans une perspective cependant plus sociale. Les travaux d'A. Chastagnol, à qui l'on doit le premier le réexamen des effets du droit latin à la lumière des études onomastiques ${ }^{68}$ ont montré que, dans certaines circonstances, les états civils enregistraient les changements de statuts intervenus à la suite d'une promotion per honorem. Le savant français avait suggéré que la filiation pérégrine pouvait être l'indice, chez ceux qui en étaient porteurs, d'une citoyenneté romaine récemment acquise. A. Chastagnol avait en outre proposé, sous certaines conditions, de considérer l'expression de la filiation par le patronyme dans les états civils de citoyens romains, comme la traduction épigraphique d'un mariage mixte intervenu à la génération des parents. Ses derniers travaux avaient envisagé la possibilité d'un aménagement du droit latin à l'époque d'Hadrien dans le but d'aligner le statut des enfants issus d'un pérégrin et d'une citoyenne romaine sur celui des enfants d'unions formées à l'inverse entre un père citoyen romain et une mère pérégrine ${ }^{69}$. L'analyse du dossier se heurtait cependant à une série de difficultés parmi lesquelles celle qui faisait observer que pour repérer des cas de promotion ob honorem ou de transmission de gentilice par la mère, A. Chastagnol avait essentiellement fondé sa réflexion et sa méthode sur l'observation du traitement de l'état civil dans les régions de tradition celtique qui privilégient souvent la filiation par le cognomen paternel plutôt que

66 Edmondson,FamilyRelations, p. 187: «Although their method has elicited some criticism, it still retains validity if we remember that its aim was to elucidate family relations rather than family structures. Some of Saller and Shaw's critics have misrepresented the goal of their study. They were clear that their figures did not allow them to reconstruct precise family structures, still less the shape and size of households; for them funerary commemorations reflected in a necessarily approximate manner the most important emotional ties that lay at the heart of family relations. »

$67 A E$, I986, 333.

68 A. Chastagnol, La Gaule romaine et le droit latin, Lyon, I995.

69 A. Chastagnol, "La condition des enfants issus de mariages inégaux entre citoyens romains et pérégrines dans les cités provinciales de droit latin », in Epigrafia romana in area adriatica, IXe Rencontre franco-italienne sur l'épigraphie du monde romain, Macerata, Io-II novembre I995, Pise-Rome, I998, p. 249-262. 
par le praenomen ${ }^{70}$. Au niveau supérieur aux élites locales, l'attention prêtée aux unions aristocratiques, près d'un siècle après les travaux de Friedrich Münzer et ceux de Ronald Syme, s'est récemment enrichie des progrès des enquêtes prosopographiques ${ }^{71}$ qui permettent de rouvrir le dossier des alliances et des désunions politiques $^{72}$ ou de s'interroger sur un aspect mal connu, celui des mésalliances ou des mariages arrangés, notamment entre chevaliers et filles de l'ordre sénatorial, quand il est possible de les repérer ${ }^{73}$.

\section{Vers une histoire globale?}

\section{Le désintérêt initial des études de genre}

Si le mariage romain n’a jamais cessé d'être un objet de curiosité, il faut bien constater que les gender studies ont au début de leur acclimatation en France peu contribué à une étude renouvelée de la question matrimoniale romaine dans la mesure où, nées dans des contextes socioculturels précis, le milieu de la psychologie au départ, ces études se sont d'abord intéressées à des catégories (amour, érotisme, identités sexuelles) ou à des expressions minoritaires ou jugées déviantes par rapport à la norme qui ne trouvaient pas immédiatement leur place dans la définition du conubium. La revue Clio sous-titrée Femmes, Genre, Histoire, fondée en I985, n’a par exemple jamais ouvert ses pages à une étude proprement dite sur le matrimonium. L'ouvrage collectif l'Histoire des femmes en Occident regrette dans l'introduction de sa seconde édition de ne pas avoir été assez une histoire du

70 P. Le Roux, Le Haut-Empire romain en Occident d'Auguste aux Sévères (3I av. J.-C.-235 ap. J.-C.), Paris, I998, p.33o; J. Gascou, “Hadrien et le droit latin », ZPE I27 (I999), p. 294-30o; $\mathrm{S}$. Armani, «Les pérégrins dans les cités romaines de Lusitanie aux deux premiers siècles de notre ère: identités et pratiques onomastiques », in J.-G. Gorges, T. Nogales Basarrate (éd.), Naissance de la Lusitanie romaine ( $I^{e r} a v .-I^{e r} a p . J .-C$.). VII Table ronde internationale sur la Lusitanie romaine, Toulouse-Mérida, 20ı, p.308-3ı2.

71 Par exemple: M.-Th. Raepsaet-Charlier, Prosopographie des femmes de l'ordre sénatorial $\left(I^{e r}-I^{e}\right.$ s. $)$, Louvain, 1987 .

72 En particulier les analyses chronologiques de M. CANAs, “Octavien, Agrippa et Atticus. La place des alliances matrimoniales dans la consolidation de la faction d'un dynaste», in R.Baudry, S.Destephen (éd.), La société romaine et ses élites. Mélanges offerts à Elizabeth Deniaux, Paris, 2012, p. I57-165 ou encore M. Canas, “À propos de la date de quelques mariages de sénateurs romains », in M.L. CALDELLI, G.L. GREGori (a cura di), Epigrafia e ordine senatorio, 30 anni dopo, Rome, 2014 (Tituli, Io), p.73-84.

73 S. Demougin, "Clarissima versus egregius: remarques sur les mariages inégaux», in M. L. Caldelli, G. L. Gregori (a cura di), Epigrafia, p. 99-IIo. 
“genre ${ }^{74}$ », dont pourtant il se revendiquait pour mieux comprendre «les relations entre les sexes, non pas inscrits dans l'éternité d'une introuvable nature, mais produits d'une construction sociale qu'il importe justement de déconstruire ${ }^{75}$ ». C'est précisément cette image du mariage romain qu'essaie de décrypter dans l'un de ses chapitres le premier manuel écrit en français sur ces questions intitulé Hommes et femmes dans l'Antiquité romaine ${ }^{76}$. Les Annales ont également tardé à intégrer ce thème à leur sommaire: dans le numéro de juillet-septembre 2012 consacré pour la première fois au genre sous un titre Régimes de genre qui entend prendre ses distances, le mariage n'est pas le thème retenu pour servir d'étude de cas à la période romaine. C'est même un autre type de situation, strict négatif du mariage romain, qui sert d'illustration ${ }^{77}$. L'article confié à une collègue allemande, Elke Hartmann, prend à contre-pied les rapports attendus de genre qui s'opèrent au sein du conubium pour montrer comment une autre hiérarchie fondée sur la distinction des sexes fonctionne aussi à l'intérieur des rapports sociaux. L'auteur y envisage en effet les relations entre corps féminin et genre, du point de vue de la succession patrimoniale en analysant un cas concret ou devenu proverbial voire caricatural, rapporté par les poètes, celui de la captation des héritages de femmes âgées, célibataires et sans enfant, l'exact contraire des matrones. Elle y montre comment ces femmes, investies d'un pouvoir inédit, celui que leur apportent la richesse et l'esprit de convoitise de leurs jeunes amants, font preuve d'une sexualité débridée digne de celle que l'on attend d'une meretrix, contraire en tout cas à leur âge et à leur rang.

\section{Reconsidérations et nouvelles perspectives}

Il a fallu attendre la fin des années 2000 pour assister au retour en grâce des études relatives au mariage avec la récente publication de l'ouvrage de Karen K.Hersch qui s'inscrit dans la lignée des études de genre. Dans The Roman $W_{e d d i n g}{ }^{78}$, la filiation historiographique est claire: le livre veut comprendre un paradoxe en réévaluant, dans le sillage d’une étude de John Scheid qui réappréciait

74 M. Perrot, “Avertissement», in G. Duby, M. Ревrot, Histoire des femmes en Occident. I. L’Antiquité (sous la direction de P. Sснмitt Pantel), 2002 (2 éd.), p. 7-8.

Perrot, Histoire des femmes, p. I9.

76 S. Boenringer, V. Sebillote Cuchet, Hommes et femmes dans l'Antiquité romaine. Le genre: méthode et documents, Paris, 20II, p. I22-125.

77 E. Hartmann, «Femmes riches et captateurs d'héritage à Rome durant le Haut-Empire », Annales. HSS 3 (2012), p.605-628.

78 Déjà cité à la note 6 . 
le rôle des femmes dans certaines fêtes religieuses ${ }^{79}$, la signification et la dimension essentiellement féminine d'une cérémonie et de rites où les femmes tenaient le premier rôle. Ce qui fait écrire à Karen Hersch que le mariage romain est une cérémonie centrée exclusivement sur l'épouse. On ne peut achever cette mise en perspective historiographique sans évoquer la très récente et stimulante étude de Marine Bretin-Chabrol ${ }^{80}$. Partant du constat que le lexique latin de la parenté file la métaphore du monde végétal, elle essaie de révéler l'idéologie sous-jacente du vocabulaire latin qui livre une lecture “genrée » de la société romaine en pensant le «mariage sur le modèle d'une coopération complémentaire entre la vigne et le support vivant d'un arbre », en l'occurrence l'orme. La recherche s'ancre dans un double courant, celui des études sur le genre dont les travaux de l'auteur se réclament explicitement ${ }^{81}$ et celui qui touche à l'histoire culturelle et des représentations. Les termes maritus, maritare désignent aussi bien l'époux que l'arbre marié à la vigne, l'action de se marier du point de vue strictement masculin que celle qui consiste à tutorer la vigne. Pour M. Bretin-Chabrol qui met en relation d'une part, l'acception tardive de maritus dans un sens strictement statutaire (l'homme marié par opposition au célibataire ou au veuf), qu'elle date précisément des lois matrimoniales d'Auguste entre ı8 a. C. et 9 p. C., et d'autre part, la technique exclusivement italienne de la vigne arbustive qui caractérise le paysage péninsulaire, seraient ici réunis tous les éléments discursifs de l'idéologie augustéenne chantée par Virgile et Horace, du retour à la paix, à la prospérité et au mos maiorum ${ }^{82}$. Pour

79 J. ScheId, “D’indispensables "étrangères”. Les rôles religieux des femmes à Rome », in Duby, Perrot, Histoire des femmes, p. 495-536, complété par J. Scheid, “Les rôles religieux des femmes à Rome. Un complément", in R.Frei-Stolba, A. Bielman, O.Bianchi, Les femmes antiques entre sphère privée et sphère publique, Berne, 2003, p. I37-I5I.

80 Bretin-Chabrol, L'arbre et la lignée. Cité à la note 2I.

81 M. Bretin-Chabrol, Cl. Leduc, “La botanique antique et la problématique du genre», Clio 29 (2009), p. 205-223.

82 Bretin-Chabrol, L'arbre et la lignée, p. 209: “L'existence, dans la langue rustique, d'une image matrimoniale désignant une telle pratique permet ensuite aux auteurs de faire de ce décor le cadre privilégié d'un mariage conçu comme proprement romain, conforme à l'idéologie du mos maiorum. L'union de la vigne et de l'orme (Virgile, Géorgique, 2, 367-37o), modèle "naturel" offert à la réflexion de ceux qui veulent réformer l'institution, est mise au service d'un discours polémique sur la meilleure forme de mariage qu'une cité doit adopter...»; p.2I7: “Lorsqu'en revanche Horace s'approprie cette image, il lui donne un contenu idéologique. Avec la bataille d'Actium et le rétablissement de la paix par Auguste s'ouvre une nouvelle ère pour les Romains qui peuvent enfin espérer retrouver la vie paisible dont la vie paisible parait les temps anciens. La prospérité du paysan enfin autorisé à cultiver ses terres sans risques doit s'accompagner de la restauration d'un foyer honnête: une épouse fidèle, des enfants légitimes. Les deux thèmes sont associés plusieurs fois chez Horace... ». 
séduisantes et convaincantes qu'elles soient, les conclusions auxquelles aboutit le livre de M. Bretin-Chabrol attire notre attention sur un point: le cloisonnement de nos disciplines. Un exemple seulement: M.Bretin-Chabrol observe que la spécialisation du terme maritus ne serait intervenue qu'après absorption du terme dans le champ institutionnel où il ne désignait plus que l'homme marié. Or, l'éclairage épigraphique révèle que non seulement, maritus est synonyme de uir et de coniux dans les épitaphes où il est employé comme terme d'adresse ou de référence, mais qu'en outre son usage a fini par susciter la création dans le langage au moins funéraire d'un symétrique féminin, la marita, attestée en grand nombre notamment en Italie, en Afrique et dans la péninsule Ibérique, ce qui contribue à nuancer les conclusions relatives à la limitation de son emploi.

L'historiographie du mariage romain s'est diversifiée au fur et à mesure que de nouveaux objets d'étude traversaient le champ d'intérêt des spécialistes de la famille et de la parenté, au fur et à mesure aussi que de nouveaux questionnements parfois inspirés par le présent s'imposaient à eux. C'est ainsi que leur regard s'est progressivement déplacé vers de nouveaux horizons géographiques et sociaux grâce aux progrès, entre autres, de la science épigraphique qui a acquis ses lettres de noblesse dans ce domaine aussi, sans en épuiser l'intérêt comme le montrent les développements récents de l'historiographie qui n'hésite pas à rouvrir d'anciens dossiers. L'écueil à éviter aujourd'hui me semble résider dans la spécialisation des études. Pour indispensables qu'elles soient, les mises au point fondées sur le droit ou les témoignages littéraires ne tiennent pas suffisamment compte de l'enseignement de l'épigraphie et ne nous font connaître que les us et coutumes des sphères supérieures, principalement romaines et italiennes. Inversement, les travaux fondés sur les seules données épigraphiques sont tributaires de la nature de leurs sources forcément limitée (contraintes dues aux catégories sociales représentées, contraintes liées aux types d'inscription, etc.). L'association des différentes perspectives me semble désormais la seule démarche susceptible de compléter les divers points de vue en exploitant la possibilité qui existe désormais d'embrasser, grâce à la multiplication des travaux, toutes les sources à notre disposition.

\section{Sabine Armani}

Maître de conférences d'histoire romaine, Université Paris 13 Sorbonne-Paris-Cité ufr llshs

99 avenue J.-B. Clément 93430 Villetaneuse sabine.armani@univ-paris13.fr 